\title{
Moving boundary shallow water flow above parabolic bottom topography
}

\author{
Joe Sampson* $^{*} \quad$ Alan Easton $^{\dagger} \quad$ Manmohan Singh $^{\ddagger}$
}

(received 16 October 2005; revised 18 August 2006)

\begin{abstract}
Exact solutions of the two dimensional nonlinear shallow water wave equations for flow involving linear bottom friction and with no forcing are found for flow above parabolic bottom topography. These solutions also involve moving shorelines. The motion decays over time. In the solution of the three simultaneous nonlinear partial differential shallow water wave equations it is assumed that the velocity is a function of time only and along one axis. This assumption reduces
\end{abstract}

* Mathematics Discipline, Faculty of Engineering and Industrial Sciences, Swinburne University of Technology, Melbourne, Australia. mailto: jsampson@swin. edu . au

${ }^{\dagger}$ Mathematics, Statistics and Computer Science Discipline, School of Natural and Physical Sciences, University of Papua New Guinea, Port Moresby, Papua New Guinea. mailto:alan.easton@upng.ac.pg; Mathematics Discipline, Faculty of Engineering and Industrial Sciences, Swinburne University of Technology, Melbourne, Australia.

$\ddagger$ Mathematics Discipline, Faculty of Engineering and Industrial Sciences, Swinburne University of Technology, Melbourne, Australia. mailto:msingh@swin.edu .au

See http://anziamj . austms.org.au/V47EMAC2005/Sampson for this article, (c) Austral. Mathematical Soc. 2006. Published October 16, 2006. ISSN 1446-8735 
the three simultaneous nonlinear partial differential equations to two simultaneous linear ordinary differential equations. The solutions found are useful for testing numerical solutions of the nonlinear shallow water wave equations which include bottom friction and whose flow involves moving shorelines.

\section{Contents}

1 Introduction

C374

2 Thacker's solutions

C375

3 Model equations

C378

4 Flow above parabolic bottom topography C379 4.1 Flow for $\tau<p \ldots \ldots \ldots \ldots \ldots \ldots \ldots$ C $38 \ldots \ldots$

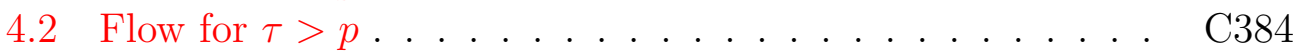

4.3 Flow for $\tau=p \ldots \ldots \ldots \ldots \ldots \ldots$ C $\ldots \ldots \ldots 4$

5 Conclusions

C385

References

C386

\section{Introduction}

Exact solutions of the nonlinear shallow water wave equations were found by Thacker [6] for frictionless flow involving the Coriolis force in parabolic canals. The solutions involve moving shorelines. The motion is oscillatory and continues indefinitely over time.

Our work builds on the work of Thacker [6]. As far as we are aware there 
have been no other analytical solutions of the nonlinear shallow water wave equations as a consequence of the work of Thacker [6] apart from an article by Sampson, Easton \& Singh [5]. Balzano [1], Holdahl, Holden \& Lie [2], Lewis \& Adams [3], Peterson, Hauser, Thacker \& Eppel [4] and Yoon \& Cho [8] compared numerical solutions of the nonlinear shallow water wave equations with some of the analytical solutions in Thacker [6].

Here exact solutions of the two dimensional nonlinear shallow water wave equations for flow involving linear bottom friction and without Coriolis force are found for flow above parabolic bottom topography. These solutions also involve moving shorelines. The motion decays over time.

\section{Thacker's solutions}

Thacker [6] considered the case where the motion of shallow water in a basin is governed by the equations [7]

$$
\begin{aligned}
& \frac{\partial U}{\partial t}+U \frac{\partial U}{\partial x}+V \frac{\partial U}{\partial y}-f V+g \frac{\partial \zeta}{\partial x}=0, \\
& \frac{\partial V}{\partial t}+U \frac{\partial V}{\partial x}+V \frac{\partial V}{\partial y}+f U+g \frac{\partial \zeta}{\partial y}=0, \\
& \frac{\partial \zeta}{\partial t}+\frac{\partial(h+\zeta) U}{\partial x}+\frac{\partial(h+\zeta) V}{\partial y}=0,
\end{aligned}
$$

where $\zeta(x, y, t)$ is the height of the water surface above mean water level, $z=-h(x, y)$ is the bottom surface, $U(x, y, t)$ is the depth averaged velocity component of the water current to the East, $V(x, y, t)$ is the depth averaged velocity component of the water current to the North, $g$ is the acceleration due to gravity, $f$ is the Coriolis parameter and $t$ is the time.

Thacker assumed that

$$
U=u_{0}(t),
$$




$$
V=v_{0}(t) .
$$

It can be shown that equations (1) and (2) together with equations (4) and (5) imply that

$$
\zeta(x, y, t)=\zeta_{0}(t)+x \zeta_{1}(t)+y \zeta_{2}(t),
$$

where

$$
\begin{aligned}
& \zeta_{1}(t)=-\frac{1}{g}\left(\frac{d u_{0}(t)}{d t}-f v_{0}(t)\right), \\
& \zeta_{2}(t)=-\frac{1}{g}\left(\frac{d v_{0}(t)}{d t}+f u_{0}(t)\right) .
\end{aligned}
$$

Thacker assumed that flow takes place in the parabolic canal

$$
h=h_{0}\left(1-\frac{x^{2}}{a^{2}}\right),
$$

with $h_{0}$ and $a$ constant.

Substituting (4) and (5) in (3) gives

$$
\frac{d \zeta_{0}(t)}{d t}+x \frac{d \zeta_{1}(t)}{d t}+y \frac{d \zeta_{2}(t)}{d t}-\frac{2 u_{0}(t) h_{0} x}{a^{2}}+u_{0}(t) \zeta_{1}(t)+v_{0}(t) \zeta_{2}(t)=0 .
$$

Equating the time-varying coefficients of the linearly independent terms 1 , $x$ and $y$ leads to

$$
\begin{aligned}
& \frac{d \zeta_{0}(t)}{d t}+u_{0}(t) \zeta_{1}(t)+v_{0}(t) \zeta_{2}(t)=0, \\
& \frac{d \zeta_{1}(t)}{d t}-\frac{2 u_{0}(t) h_{0}}{a^{2}}=0 . \\
& \frac{d \zeta_{2}(t)}{d t}=0 .
\end{aligned}
$$

Substituting (7) in (12) gives

$$
\frac{d^{2} u_{0}(t)}{d t^{2}}-f \frac{d v_{0}}{d t}+\frac{2 g h_{0} u_{0}(t)}{a^{2}}=0 .
$$


Substituting (8) in (13) gives

$$
\frac{d^{2} v_{0}(t)}{d t^{2}}+f \frac{d u_{0}}{d t}=0
$$

Substituting (7) and (8) in (11) gives

$$
\frac{d \zeta_{0}(t)}{d t}-\frac{1}{g} u_{0}(t) \frac{d u_{0}}{d t}-\frac{1}{g} v_{0}(t) \frac{d v_{0}}{d t}=0 .
$$

Thacker gave solutions to equations (14), (15) and (16) without explaining how he obtained his solutions. We discuss below how one could obtain Thacker's solutions. If one differentiates (14) with respect to $t$ and makes use of (15) one obtains a third order differential equation for $u_{0}(t)$ :

$$
\frac{d^{3} u_{0}(t)}{d t^{3}}+\left(f^{2}+\frac{2 g h_{0}}{a^{2}}\right) \frac{d u_{0}(t)}{d t}=0 .
$$

A solution is

$$
u_{0}(t)=-G \Omega \sin (\Omega t),
$$

where $G$ is a constant and

$$
\Omega=\left(f^{2}+\frac{2 g h_{0}}{a^{2}}\right)^{1 / 2}
$$

Substitution of (18) in (15) gives the solution

$$
v_{0}(t)=-G f \cos (\Omega t) .
$$

Substitution of (18) and (20) in (16) and then integration with respect to $t$ gives the solution

$$
\zeta_{0}(t)=-\frac{G^{2} h_{0}}{a^{2}} \cos ^{2}(\Omega t)
$$

Substitution of (18) and (20) in (7) gives

$$
\zeta_{1}(t)=\frac{2 G h_{0}}{a^{2}} \cos (\Omega t) .
$$


Substitution of (18) and (20) in (7) gives

$$
\zeta_{2}=0 \text {. }
$$

Substitution of (21) and (22) and (23) in (6) gives

$$
\zeta(x, y, t)=-\frac{G^{2} h_{0}}{a^{2}} \cos ^{2}(\Omega t)+\frac{2 G h_{0}}{a^{2}}(\cos (\Omega t)) x .
$$

At the shoreline the total depth is

$$
h+\zeta=0 .
$$

Substitution of (9) and (24) in (25) gives the shorelines

$$
x=G \cos (\Omega t) \pm a .
$$

\section{$3 \quad$ Model equations}

We consider the case where the motion of shallow water in a basin is governed by the equations $[7]$

$$
\begin{aligned}
& \frac{\partial U}{\partial t}+U \frac{\partial U}{\partial x}+V \frac{\partial U}{\partial y}+\tau U+g \frac{\partial \zeta}{\partial x}=0 \\
& \frac{\partial V}{\partial t}+U \frac{\partial V}{\partial x}+V \frac{\partial V}{\partial y}+\tau V+g \frac{\partial \zeta}{\partial y}=0 \\
& \frac{\partial \zeta}{\partial t}+\frac{\partial(h+\zeta) U}{\partial x}+\frac{\partial(h+\zeta) V}{\partial y}=0
\end{aligned}
$$

where $\tau$ is the bottom friction parameter, $g$ is the acceleration due to gravity and $t$ is the time. The bottom friction parameter, $\tau$, is considered to be constant.

Equations (27), (28) and (29) differ from Thacker's equations in that whereas Thacker's equations included Coriolis force terms ( see Section 2) 
for Thacker's solutions) but did not include friction terms, equations (27), (28) and (29) do not include Coriolis force terms, but do include friction terms.

Thacker [6] assumed that $U$ and $V$ were functions of $t$ only. Here we assume

$$
\begin{aligned}
& U=u_{0}(t), \\
& V=0,
\end{aligned}
$$

Then equations (27) and (28) together with equations (30) and (31) imply

$$
\zeta(x, y, t)=\zeta_{0}(t)+x \zeta_{1}(t)
$$

where (27) and (30) imply

$$
\zeta_{1}(t)=-\frac{1}{g}\left(\frac{d u_{0}(t)}{d t}+\tau u_{0}(t)\right)
$$

It will be shown later how $\zeta_{0}(t)$ is determined. Equation (32) shows that at any time $t$ the water surface is a plane.

We consider flows above parabolic bottom topography. The discussion in Section 4 is similar to that in Thacker [6], but because the shallow water equations (27) and (28) used in this article have a slightly different form to Thacker's and we make slightly different assumptions about the velocity's functional form, the discussion leads to different conclusions.

\section{Flow above parabolic bottom topography}

Following Thacker, assume that

$$
h=h_{0}\left(1-\frac{x^{2}}{a^{2}}\right),
$$


with $h_{0}$ and $a$ constant, so that flow takes place above parabolic bottom topography.

Substituting (30), (31), (32), and (34) in (29) gives

$$
\frac{d \zeta_{0}(t)}{d t}+x \frac{d \zeta_{1}(t)}{d t}-\frac{2 u_{0}(t) h_{0} x}{a^{2}}+u_{0}(t) \zeta_{1}(t)=0 .
$$

Following Thacker, equate the time-varying coefficients of the linearly independent terms, 1 and $x$ respectively,

$$
\begin{aligned}
& \frac{d \zeta_{0}(t)}{d t}+u_{0}(t) \zeta_{1}(t)=0 \\
& \frac{d \zeta_{1}(t)}{d t}-\frac{2 u_{0}(t) h_{0}}{a^{2}}=0 .
\end{aligned}
$$

Substituting (33) in (37)

$$
\frac{d^{2} u_{0}(t)}{d t^{2}}+\tau \frac{d u_{0}(t)}{d t}+\frac{2 g h_{0} u_{0}(t)}{a^{2}}=0 .
$$

Substituting (33) in (36)

$$
\frac{d \zeta_{0}(t)}{d t}-\frac{1}{g} u_{0}(t) \frac{d u_{0}}{d t}-\frac{\tau}{g} u_{0}(t)^{2}=0 .
$$

Equation (38) has to be solved for $u_{0}(t)$.

As equation (38) is a second order differential equations, it requires two boundary conditions. The solution of (38) can be substituted in (39), which is first order and hence needs one boundary condition to be solved uniquely for $\zeta_{0}(t)$.

The auxiliary equation for (38) is

$$
\lambda^{2}+\tau \lambda+\frac{2 g h_{0}}{a^{2}}=0 .
$$


The roots of (40) are

$$
\lambda=\frac{-\tau \pm \sqrt{\tau^{2}-p^{2}}}{2} .
$$

where

$$
p=\sqrt{\frac{8 g h_{0}}{a^{2}}} .
$$

Hence, the three possible solutions of (40) are for when $\tau\langle p, \tau\rangle p$, and $\tau=p$. Consideration of some typical values of $a, h_{0}$ and $\tau$ shows that all the possible solutions are realistic solutions of (40). Thus there are three realistically possible solutions of (38). The solutions of (32), (36) and (38) for $\tau<p, \tau>p$ and $\tau=p$ are discussed in the following three subsections.

\subsection{Flow for $\tau<p$}

If $\tau<p$, then a solution of (38) is

$$
u_{0}(t)=B e^{-\tau t / 2} \sin s t
$$

where $B$ is a constant, obtained by using given values for $u_{0}(0)$ and $u_{0}^{\prime}(0)$, and where

$$
s=\frac{\sqrt{p^{2}-\tau^{2}}}{2} .
$$

It can be seen from (43) that as $t \rightarrow \infty, u_{0}(t) \rightarrow 0$.

Substituting (43) in (39) and integrating with respect to $t$ gives

$$
\zeta_{0}(t)=\frac{a^{2} B^{2} e^{-\tau t}}{8 g^{2} h_{0}}\left(-s \tau \sin 2 s t+\left(\frac{\tau^{2}}{4}-s^{2}\right) \cos 2 s t\right)-\frac{B^{2} e^{-\tau t}}{4 g},
$$

with the constant of integration being zero because it is assumed that as $t \rightarrow \infty, \zeta_{0}(t) \rightarrow 0$. 
Substituting (43) in (33)

$$
\zeta_{1}(t)=-\frac{e^{-\tau t / 2}}{g}\left(B s \cos s t+\frac{\tau B}{2} \sin s t\right) .
$$

Substituting (45) and (46) into (32)

$$
\begin{aligned}
\zeta(x, t)= & \frac{a^{2} B^{2} e^{-\tau t}}{8 g^{2} h_{0}}\left(-s \tau \sin 2 s t+\left(\frac{\tau^{2}}{4}-s^{2}\right) \cos 2 s t\right)-\frac{B^{2} e^{-\tau t}}{4 g} \\
& -\frac{e^{-\tau t / 2}}{g}\left(B s \cos s t+\frac{\tau B}{2} \sin s t\right) x .
\end{aligned}
$$

It can be seen that as $t \rightarrow \infty, \zeta(t) \rightarrow 0$; that is, the displacement of the fluid from equilibrium gradually dies out over time, which is the result that one would expect with a bottom friction force acting on the fluid.

At the shoreline, the total depth

$$
h+\zeta=0 .
$$

Substituting (34) and (47) in (48) gives

$$
\left(x-\frac{a^{2} e^{-\tau t / 2}}{2 h_{0} g}\left(-B s \cos s t-\frac{\tau B}{2} \sin s t\right)\right)^{2}=a^{2} .
$$

Hence, the projection of the moving shorelines on the $x y$ plane is two parallel straight lines:

$$
x=\frac{a^{2} e^{-\tau t / 2}}{2 h_{0} g}\left(-B s \cos s t-\frac{\tau B}{2} \sin s t\right) \pm a .
$$

The water moves backwards and forwards across the canal with motion dying out as $t \rightarrow \infty$. As $t \rightarrow \infty$ the shorelines approach

$$
x= \pm a,
$$



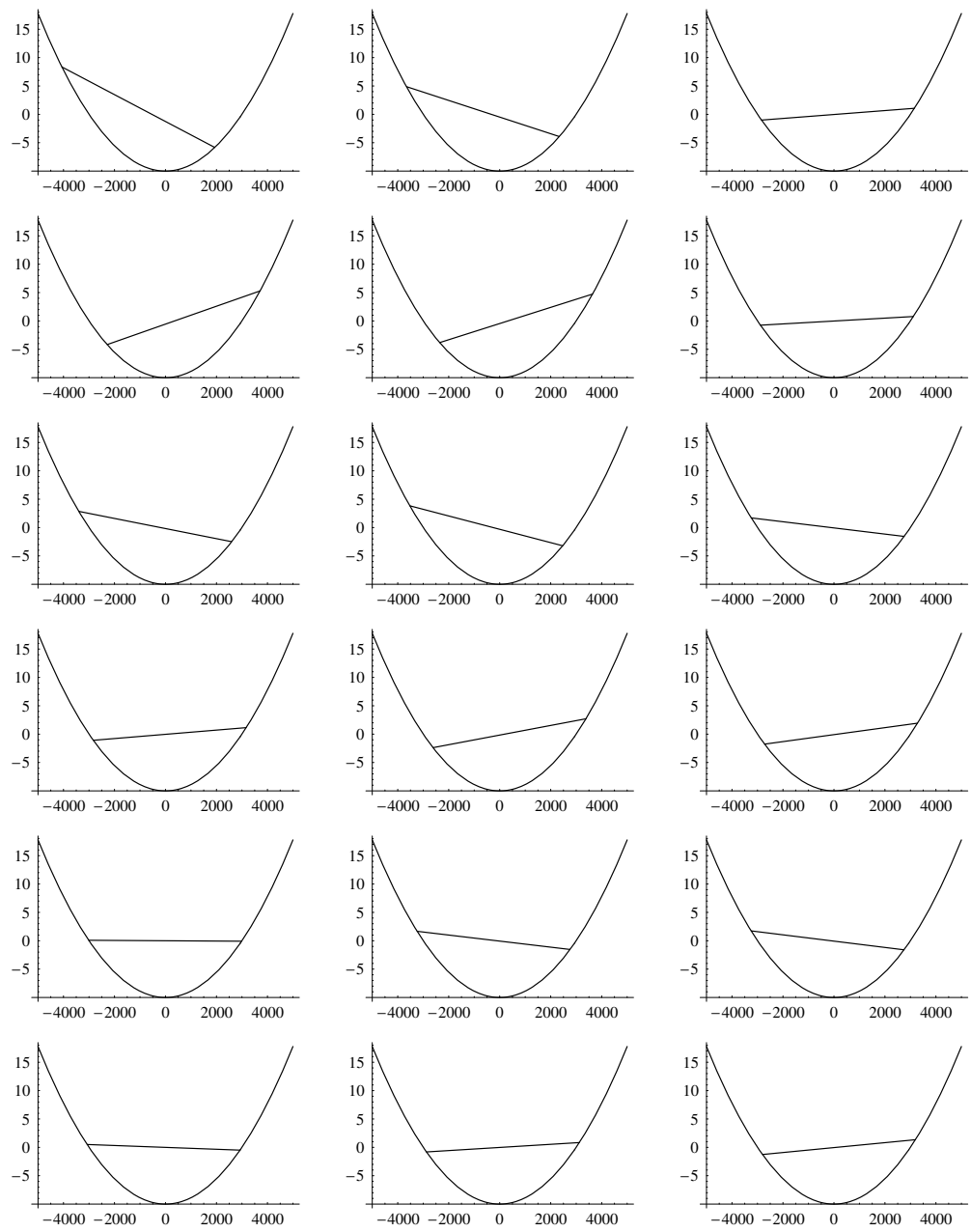

Figure 1: The development of the motion of fluid in a parabolic canal for which $a=3 \mathrm{~km}, h_{0}=10 \mathrm{~m}$, and $\tau=0.001 \mathrm{~s}^{-1}$, for motion in which $B=5$ $\mathrm{ms}^{-1}$, from $t=0 \mathrm{~s}$ to $t=3400 \mathrm{~s}$, in increments of $200 \mathrm{~s}$. Dimensions are in metres on the vertical axis and on the horizontal axis. 
the shorelines for an undisturbed surface, and $\zeta \rightarrow 0$, so that friction will cause the initial disturbance to eventually die out.

Consider a canal for which $a=3 \mathrm{~km}, h_{0}=10 \mathrm{~m}$, and $\tau=0.001 \mathrm{~s}^{-1}$, for motion in which $B=5 \mathrm{~m} \mathrm{~s}^{-1}$. The development of the motion from $t=0 \mathrm{~s}$ to $t=3400 \mathrm{~s}$, in increments of $200 \mathrm{~s}$ is shown in Figure 1.

\subsection{Flow for $\tau>p$}

When $\tau>p$ then a solution of (38) is

$$
u_{0}(t)=B e^{r t},
$$

where $B$ is a constant, obtained by using given values for $u_{0}(0)$ and $u_{0}^{\prime}(0)$, and where

$$
r=\frac{-\tau-\sqrt{\tau^{2}-p^{2}}}{2}
$$

It can be shown that

$$
\zeta(x, t)=\frac{1}{g}\left(\left(\frac{r+\tau}{2 r}\right)\left(B^{2} e^{2 r t}\right)\right)-\frac{1}{g}(r+\tau) B e^{r t} x .
$$

The projection of the moving shoreline on the $x y$ plane is two parallel straight lines

$$
x=-\frac{a^{2}}{2 g h_{0}}\left(B(r+\tau) e^{r t}\right) \pm a .
$$

\subsection{Flow for $\tau=p$}

When $\tau=p$ then the solution of (38) is

$$
u_{0}(t)=e^{-\tau t / 2}(A+B t)
$$

where $A$ and $B$ are constants. It can be shown that

$$
\zeta(x, t)=-\frac{e^{-\tau t}}{\tau}\left(\frac{1}{g}\left(A B+\frac{\tau A^{2}}{2}\right)+\frac{1}{g}\left(B^{2}+\tau A B\right)\left(t+\frac{1}{\tau}\right)\right)
$$




$$
\begin{aligned}
& -e^{-\tau t}\left(\frac{B^{2}}{2 g}\left(t^{2}+\frac{2 t}{\tau}+\frac{2}{\tau^{2}}\right)\right) \\
& -\frac{x}{g}\left(B+\frac{\tau}{2}(A+B t)\right) e^{-\tau t / 2} .
\end{aligned}
$$

The projection of the moving shoreline on the $x y$ plane is two parallel straight lines:

$$
x=-\frac{a^{2}}{2 g h_{0}} e^{-\tau t / 2}\left(B+\frac{\tau}{2}(A+B t)\right) \pm a .
$$

\section{Conclusions}

Exact solutions of the two dimensional nonlinear shallow water wave equations in the case of flow involving bottom friction and without the Coriolis force have been found for flow in a parabolic canal. These solutions also involve moving shorelines. The motion decays over time, which is what one would expect in a motion involving friction and no input force. In contrast, Thacker found exact solutions of the two dimensional nonlinear shallow water wave equations in the case of flow involving the Coriolis force but without bottom friction for flow in a parabolic canal. These solutions also involve moving shorelines. The motion is oscillatory and continues indefinitely over time, which is what one would expect in a motion involving no friction.

The solutions found in this paper are useful for testing numerical solutions of the nonlinear shallow water wave equations which include bottom friction and whose flow involves moving shorelines. Testing of the analytical solutions against two numerical schemes showed the numerical results to be in close agreement with the analytical solutions. 


\section{References}

[1] Balzano, A. Evaluation of methods for numerical simulation of wetting and drying in shallow water flow models. Coastal Engineering. 34-2, 1, 1998, 83-107. C375

[2] Holdahl, R., Holden, H., and Lie, K-A. Unconditionally stable splitting methods for the shallow water equations, BIT , 39, 1998, 451-472. C375

[3] Lewis, C. H. III and Adams, W. M. Development of a tsunami-flooding model having versatile formation of moving boundary conditions. The Tsunami Society Monograph Series, 1983, No. 1, 128 pp. C375

[4] Peterson P., Hauser J., Thacker W. C., Eppel D., An error-minimizing algorithm for the non-linear shallow-water wave equations with moving boundaries. In Numerical Methods for Non-Linear Problems, editors C. Taylor, E. Hinton, D. R. J. Owen and E. Onate, 2, Pineridge Press, 1984, 826-836. C375

[5] Sampson, J., Easton, A. and Singh, M. Moving boundary shallow water flow in circular paraboloidal basins. Proceedings of the Sixth Engineering Mathematics and Applications Conference, 5th International Congress on Industrial and Applied Mathematics, at the University of Technology, Sydney, Australia, editors R. L. May and W. F. Blyth, 2003, 223-227. C375

[6] Thacker, W. C. Some exact solutions to the nonlinear shallow-water wave equations, J. Fluid. Mech., 107, 1981, 499-508. C374, C375, C379

[7] Vreugdenhil, C. B. Numerical methods for shallow-water flow, Kluwer Academic Publishers, 1998. C375, C378 
[8] Yoon S. B. and Cho J. H. Numerical simulation of coastal inundation over discontinuous topography, Water Engineering Research, 2(2), 2001, 75-87. C375 